\title{
Induction of Effector and Memory Cellular Immunity in a Patient with Long-Term Complete Molecular Response to Philadelphia Chromosome-Positive Acute Lymphoblastic Leukemia
}

\author{
Tatsuro Jo $^{a}$ Haruna Shioya $^{b}$ Hiroo Tominaga $^{b}$ Takahiro Sakai $^{b}$ \\ Shizuka Hayashi $^{b} \quad K^{2}$ aori Matsuzaka ${ }^{b}$ Yohei Kaneko $^{b}$ Masatoshi Matsuo $^{a}$ \\ Jun Taguchi ${ }^{\text {a }}$ \\ aDepartment of Hematology, Japanese Red Cross Nagasaki Genbaku Hospital, Nagasaki, \\ Japan; bepartment of Laboratory, Japanese Red Cross Nagasaki Genbaku Hospital, \\ Nagasaki, Japan
}

Keywords

Philadelphia chromosome $\cdot$ Cytotoxic T lymphocyte $\cdot$ bcr-abl1 $\cdot$ Wilms tumor 1

\begin{abstract}
This case report is about a patient who suffered from Philadelphia chromosome (Ph1)-positive acute lymphoblastic leukemia. The blasts were positive for myeloid-lineage markers including CD13 and CD33, as well as B-cell-lineage markers. Minor bcr-abl1 mRNA was detected by real-time quantitative polymerase chain reaction. Chromosomal abnormality monosomy 7 was also observed, in addition to Ph1. Despite treatment difficulties that were anticipated based on these findings, the patient had long-time complete molecular response (CMR) for approximately 5 years using chemotherapy and two tyrosine kinase inhibitors, imatinib and dasatinib. Lymphocytes were elevated after the patient switched from imatinib to dasatinib, and a T-cell receptor (TCR) $V$ beta gene repertoire analysis revealed oligoclonal expansion of effector and memory cytotoxic T lymphocytes (CTLs), including Wilms tumor 1-specific CTLs. More specifically, the two memory CTLs expressing TCR $V$ beta 3 and $V$ beta 7.1 gradually increased after dasatinib administration. The activation and maintenance of anti-leukemia immunity may have allowed the patient to obtain long-time CMR. These results highlight that obtaining memory CTLs for leukemia cells may lead to safe withdrawal from dasatinib in the patient.


Jo et al.: Cellular Immunity in Ph1-Positive Acute Lymphoblastic Leukemia

\section{Introduction}

Philadelphia chromosome ( $\mathrm{Ph} 1)$, resulting from the reciprocal translocation between chromosomes 9 and 22, can lead to the formation of major and minor bcr-abl1 chimeric genes [1]. Major bcr-abl1 is generally detected in chronic myeloid leukemia (CML), whereas minor bcr-abl1 is usually detected in $\mathrm{Ph} 1^{+}$acute lymphoblastic leukemia (ALL), a chemotherapyrefractory hematologic neoplasm [1]. Dasatinib, a second-generation tyrosine kinase inhibitor (TKI) that blocks multiple tyrosine kinases, such as BCR-ABL and Src kinases, is approved for CML and Ph1 $1^{+}$ALL [2]. Patients treated with dasatinib, but not other TKIs, often experience elevated lymphocyte counts and large granular lymphocytes $[3,4]$. This case report is of a patient with $\mathrm{Ph} 1^{+}$ALL who achieved long-term survival. The patient developed oligoclonal effector and memory cytotoxic T lymphocytes (CTLs), including Wilms tumor 1 (WT1)specific CTLs.

\section{Case Presentation}

A 54-year-old female was admitted to our hospital with leukocytosis and abnormal cells. Table 1 summarizes the laboratory data. The patient had an elevated leukocyte count $(28,400 /$ $\mu \mathrm{L})$ with $75.5 \%$ blasts; and slight thrombocytopenia was also observed $\left(11.9 \times 10^{4} / \mu \mathrm{L}\right)$. Bone marrow aspiration results were not available due to a dry tap. Flow cytometry of the peripheral mononuclear cells revealed that the blasts were positive for CD10, CD13, CD19, CD33, and CD34 and negative for CD2, CD3, CD4, CD5, CD7, CD8, CD14, CD20, CD41, CD56, and CD235a. Ph1 positivity with monosomy 7 was detected by the chromosomal analysis of peripheral blood. Messenger RNA, extracted from peripheral mononuclear cells and analyzed by realtime quantitative polymerase chain reaction (RQ-PCR), revealed that the patient was positive for minor $b c r-a b l 1$ and negative for major $b c r-a b l 1$. Based on this data, the patient was diagnosed with $\mathrm{Ph} 1^{+}$ALL. Induction chemotherapy, comprised of daunorubicin $\left(50 \mathrm{mg} / \mathrm{m}^{2}\right.$ on days $1-5)$ and cytarabine $\left(100 \mathrm{mg} / \mathrm{m}^{2}\right.$ on days $\left.1-7\right)$, was initiated. One month later, dasatinib (140 mg/day) was added after the identification of minor bcr-abl1. Complete remission was confirmed 1 month after the induction therapy, and complete molecular response (CMR, no detectable minor bcr-abl1 by RQ-PCR) was observed 2 months after the induction therapy. Therefore, the first consolidation therapy, comprised of daunorubicin $\left(45 \mathrm{mg} / \mathrm{m}^{2}\right.$ on days $1-3)$, vincristine ( $2 \mathrm{mg}$ /body on days 1 and 8 ), and prednisolone (60 mg/m² on days 1-7), was administered 1 month after induction therapy (consolidation A). The patient has maintained complete remission and CMR to date. Thereafter, a second consolidation therapy (consolidation B) round, comprised of etoposide $\left(150 \mathrm{mg} / \mathrm{m}^{2}\right.$ on days $\left.1-4\right)$ and cytarabine $\left(2,000 \mathrm{mg} / \mathrm{m}^{2}\right.$ on days $\left.1-4\right)$, was administered 2 months after induction therapy, and the dasatinib dose was reduced to $100 \mathrm{mg} /$ day 3 months after the induction therapy. The third consolidation therapy (consolidation C) round, comprised of methotrexate $\left(2,360 \mathrm{mg} / \mathrm{m}^{2}\right.$ on days 1 and 15), was administered 3 months after induction therapy. One cycle of consolidation $\mathrm{B}$ and one cycle of consolidation $\mathrm{C}$ were administered 4 and 5 months after induction therapy, respectively. Thereafter, the patient has had outpatient follow-ups.

Dasatinib was changed to the first-generation TKI imatinib (600 mg/day) 7 months after initiating therapy, due to a pleural effusion that was considered a dasatinib-associated adverse event (Fig. 1A). The imatinib dose was reduced to $400 \mathrm{mg} /$ day 9 months after initiating therapy and continued for 23 months, when it was changed to a reduced-dose dasatinib $(20 \mathrm{mg} /$ day initially and $40 \mathrm{mg}$ /day 36 months after initiating therapy) in combination with diuretics per the patient's preference. After the switch to dasatinib, the lymphocyte counts gradually increased (Fig. 1B) [3, 4]. The treatments are summarized in further detail in Figure 1. 
Table 1. Laboratory data on first admission

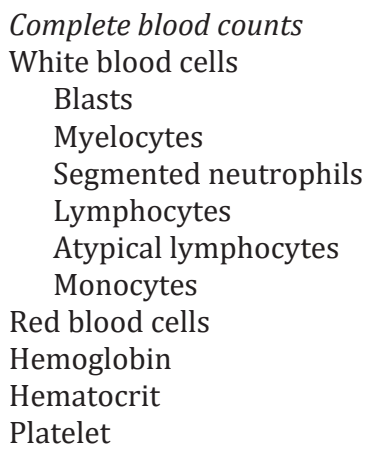

$$
\begin{aligned}
& 28,400 / \mu \mathrm{L} \\
& 75.5 \% \\
& 0.5 \% \\
& 8.5 \% \\
& 12.5 \% \\
& 1.0 \% \\
& 1.5 \% \\
& 435 \times 10^{4} / \mu \mathrm{L} \\
& 13.6 \mathrm{~g} / \mathrm{dL} \\
& 39.4 \% \\
& 11.9 \times 10^{4} / \mu \mathrm{L}
\end{aligned}
$$

Dry tap

\begin{tabular}{ll} 
& CD3: $0.1 \%$ \\
& CD4: $0.1 \%$ \\
& CD7: $3.5 \%$ \\
CD8: $0.0 \%$ \\
B-cell lineage & CD56: $0.0 \%$ \\
& CD10: $97.7 \%$ \\
Myeloid lineage/others & CD19: $99.9 \%$ \\
& CD20: $0.9 \%$ \\
& CD13: $99.6 \%$ \\
& CD33: $82.7 \%$ \\
& CD34: $99.8 \%$ \\
CD4 $1: 2.4 \%$ \\
Chromosomal analysis (peripheral blood) & CD $117: 18.0 \%$ \\
\hline bcr-abl1 mRNA analysis (RQ-PCR) & HLA-DR: $18 \%$ \\
Major bcr-abl1 mRNA & $45, X X,-7, t(9 ; 22)[4] / 46, X X[1]$ \\
\hline Minor bcr-abl1 mRNA & Not detected \\
\hline
\end{tabular}

RQ-PCR, real-time quantitative polymerase chain reaction.

T-cell receptor (TCR) $V$ beta repertoire analysis by flow cytometry using a beta mark TCR $\mathrm{V} \beta$ repertoire kit (Beckman Coulter, Tokyo, Japan) was conducted 31 months after the induction therapy. Figure 2 highlights the changes in the rates of effector and memory CTLs. Our analyses revealed two effector CTL clones, expressing TCR $V$ beta 3 and $V$ beta 17 genes, and two memory CTL clones, expressing TCR $V$ beta 3 and $V$ beta 7.1 genes. More specifically, the two memory CTL clones gradually increased after resuming dasatinib administration (Fig. 1 and 2). The patient harbored human leukocyte antigens (HLAs)-A*02:06:01 and -A*31:01:02. Some CML-specific tumor antigens, restricted by HLA-A*02:01/A*02:06:01, were also previously reported $[5,6]$. Therefore, a tetramer analysis was performed on the patient's peripheral blood. All tetramers were purchased from BML (Nagoya, Japan). Cytomegalovirus-specific CTLs and CTLs recognizing WT1 $1_{37-45}$ were detected 56 months after the induction therapy (Table 2). 


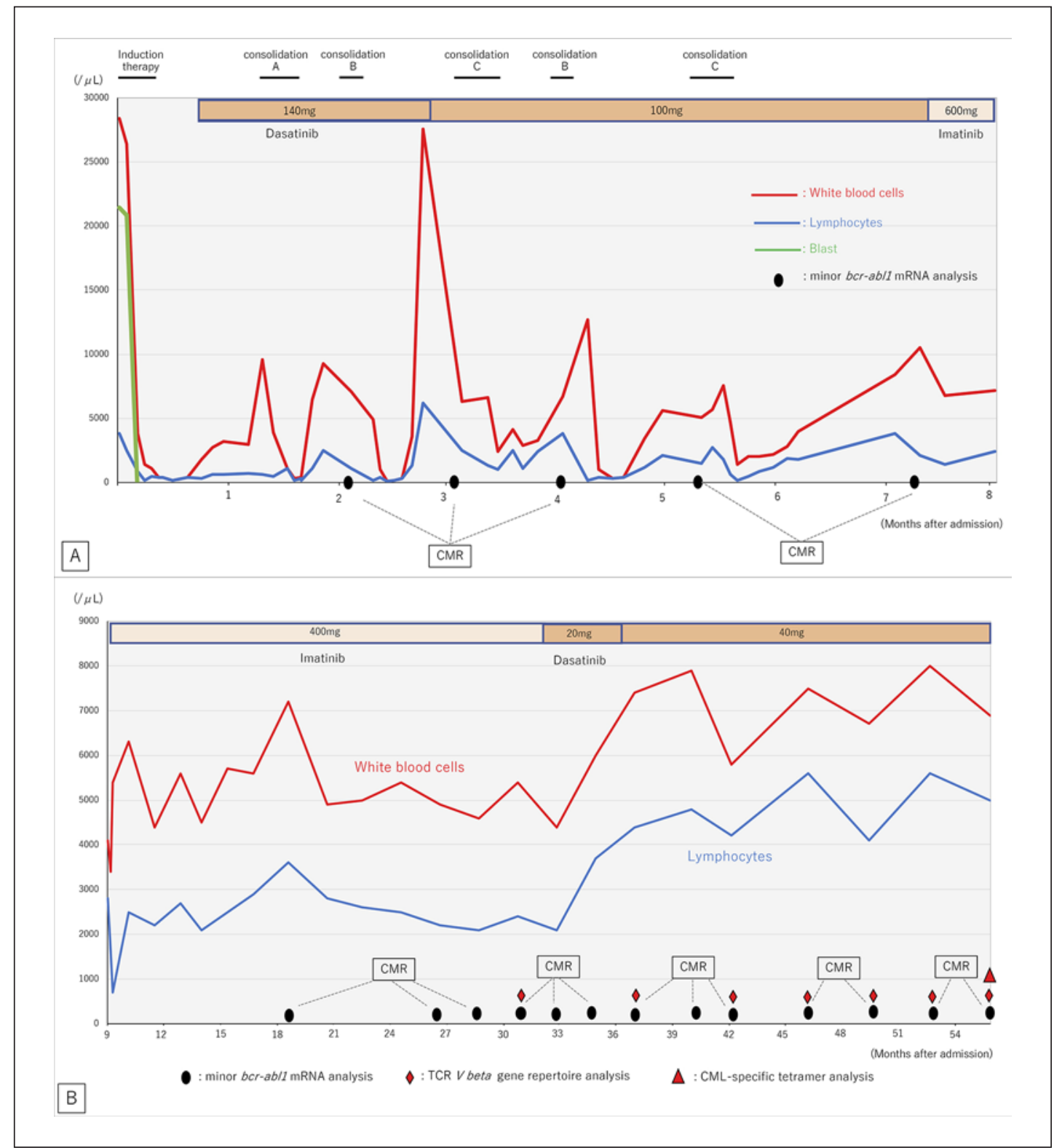

Fig. 1. The patient's clinical course, including changes in white blood cell counts, blasts, and lymphocytes; interventions; T-cell receptor (TCR) V beta gene repertoire analysis; and chronic myeloid leukemia (CML)-specific tetramer analysis. A Chemotherapy course with dasatinib/imatinib. B Follow-up course with imatinib/ dasatinib. The red, blue, and green lines indicate white blood cells, lymphocytes, and blasts, respectively. The black ovals indicate timepoints when mRNA analysis was conducted to assess minor bcr-abl1 status. The red rhombi indicate timepoints when TCR $V$ beta gene repertoire analysis was conducted. The red triangle indicates timepoint when CML-specific tetramer analysis was performed. CMR, complete molecular response.

\section{Discussion/Conclusion}

First- and second-generation TKIs have led to significant improvements in treating both CML and Ph1 ${ }^{+}$ALL. Patients with chronic phase (CP)-CML who attain deep molecular responses will have a life expectancy comparable to that of the general population [7]. However, TKIs alone cannot eradicate $\mathrm{Ph} 1^{+}$cancer stem cells [8]. Discontinuing TKI therapy to obtain 


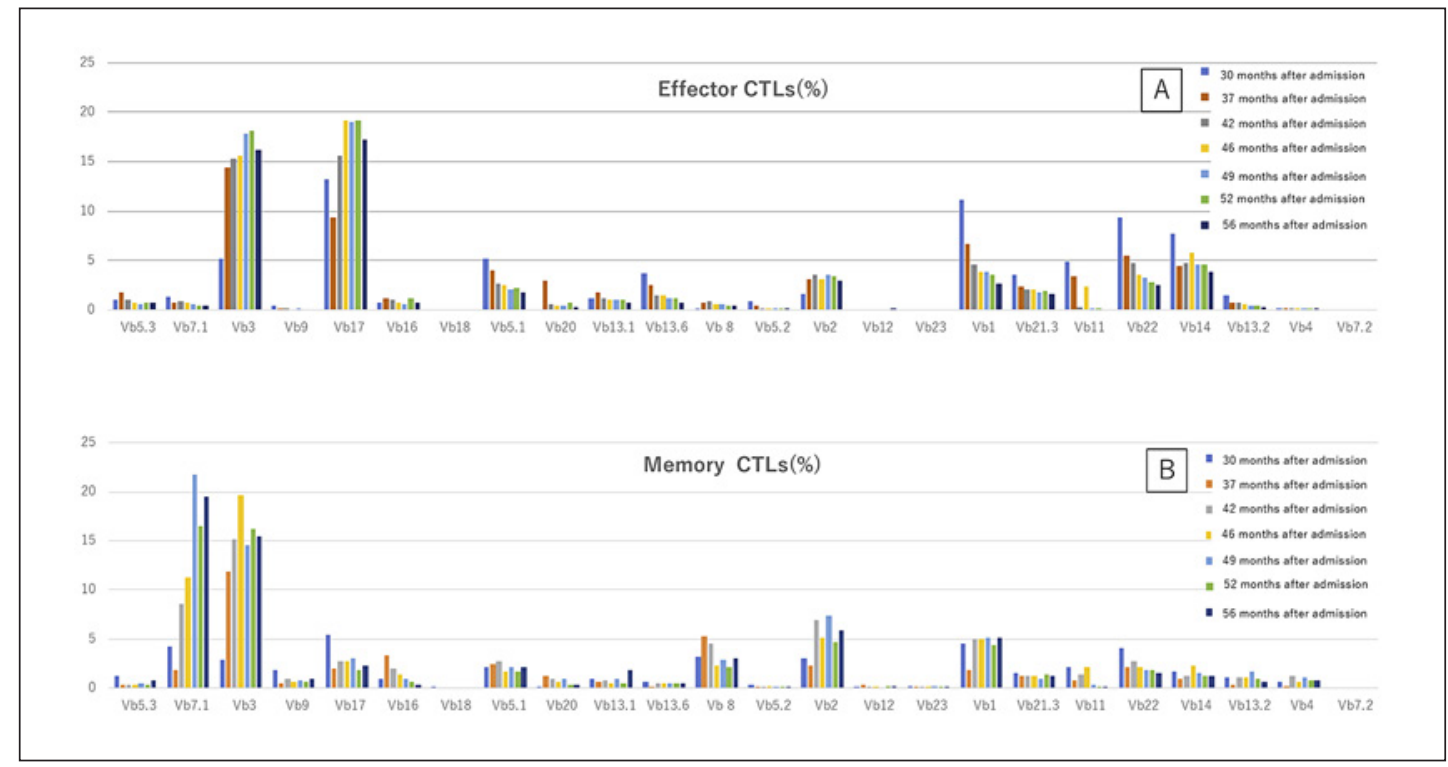

Fig. 2. T-cell receptor $V$ beta gene repertoire analysis. A Effector cytotoxic T lymphocytes (CTLs) (\%). B Memory CTLs (\%). Peripheral blood mononuclear cells were analyzed by flow cytometric analysis using the beta mark TCR V $\beta$ repertoire kit (Beckman Coulter, Tokyo, Japan) according to the manufacturer's instructions. Effector CTLs were defined as the CD8+CD27-CD45RA + population, and memory CTLs were defined as the CD8+CD45RA-population.

Table 2. Tetramer analyses

\begin{tabular}{lll}
\hline Tumor antigen & Peptides & $\begin{array}{l}\text { Cytotoxic T } \\
\text { lymphocytes } \\
(\%)\end{array}$ \\
\hline CMV & NLVPMVATV & 2.012 \\
WT1 $_{37-45}$ & VLDFAPPGA & 0.006 \\
WT1 $_{126-134}$ & RMFPNAPYL & 0.000 \\
hTERT & ILAKFLHWL & 0.000 \\
BCR-ABL & GVRGRVEEI & 0.000 \\
PR1 & VLQELNVTV & 0.000 \\
\hline
\end{tabular}

The patient's peripheral blood was directly analyzed by tetramer analysis to detect tumor antigen-specific CTLs, in the absence of stimulation. All tetramers were purchased from BML (Nagoya, Japan). CML, chronic myeloid leukemia; WT1, Wilms tumor 1; hTERT, human telomerase reverse transcriptase.

treatment-free remission (TFR) has been investigated previously in various conditions; half of all patients can maintain a deep molecular response [9]. Considering the immunological aspects related to the efficacy of interferon alpha, hematopoietic stem cell transplantation, and donor leukocyte infusion, upregulating cellular immunity in $\mathrm{Ph} 1^{+}$cancer stem cells may be deemed necessary to obtain a cure. We have recently reported on a patient with CP-CML who maintained TFR for several years after the cessation of dasatinib [10]. Maintenance of memory and effector CTLs with TCR clonality observed in the previous patient suggested that clonal expansion of CTLs against CML stem cells may be a meaningful marker of TKI cessation [10]. 
The blasts of the present patient expressed myeloid-lineage markers CD13 and CD33, as well as B-cell-lineage markers. Furthermore, the blasts harbored monosomy 7 in addition to Ph1. Although difficulties in treatment were anticipated based on these findings, the patient was able to achieve long-term CMR for approximately 5 years, using chemotherapy and TKI treatment. Cytotoxic cellular immunity, assessed by TCR $V$ beta gene repertoire analysis, revealed the oligoclonal expansion of effector and memory CTLs (Fig. 2). Interestingly, two memory CTL clones expressing TCR $V$ beta 7.1 and $V$ beta 3 gradually increased after the resumption of dasatinib 32 months after initiation therapy. The data suggests that dasatinib may play two roles: acting as a molecular-targeted therapy and activating and maintaining host cellular immunity against $\mathrm{Ph} 1^{+}$cancer stem cells. Based on our experience in a patient with CP-CML, as noted above, it may be safe to discontinue dasatinib in the present patient.

The analysis of several known tumor antigens in CML, using tetramer analysis, revealed that the present patient had WT1 $1_{37-45}$-specific CTLs. Tetramers for cytomegalovirus-specific CTLs were used as a positive control.

In summary, this case report describes a patient with Ph1 ALL, who was treated with chemotherapy and TKI treatment, and obtained long-term CMR. Dasatinib may have played an important role in activating host cellular immunity and maintaining effector and memory CTLs. These findings, coupled with our previous experience with a patient with CP-CML who achieved TFR, suggest that elevation in effector and memory CTL numbers against Ph1 ${ }^{+}$ cancer stem cells may lead to long-term CMR and TFR.

\section{Acknowledgment}

The authors would like to thank Ms. Riyo Matsumoto for editing the manuscript.

\section{Statement of Ethics}

The patient gave us written informed consent to publish the content of this manuscript including the use and disclosure of protected health information.

\section{Disclosure Statement}

The authors have no conflicts of interest to declare for this study.

\section{Funding Sources}

There was no funding received while preparing the manuscript.

\section{Author Contributions}

T.J. wrote the manuscript including designing the work and acquiring, analyzing, and interpreting the data. Y.K., K.M., H.S., T.S., H.T., and S.H. conducted the acquisition and the analysis of data. M.M., and J.T. discussed the conception of the manuscript. 


\section{References}

1 Soverini S, Mancini M, Bavaro L, Cavo M, Martinelli G. Chronic myeloid leukemia: the paradigm of targeting oncogenic tyrosine kinase signaling and counteracting resistance for successful cancer therapy. Mol Cancer. 2018;17(1):49.

2 Keating GM. Dasatinib: A review in chronic myeloid leukaemia and Ph+ acute lymphoblastic leukaemia. Drugs. 2017;77(1):85-96.

3 Mustjoki S, Ekblom M, Arstila TP, Dybedal I, Epling-Burnette PK, Guilhot F, etal. Clonal expansion of T/NK-cells during tyrosine kinase inhibitor dasatinib therapy. Leukemia. 2009;23(8):1398-405.

4 Kim DH, Kamel-Reid S, Chang H, Sutherland R, Jung CW, Kim HJ, et al. Natural killer or natural killer/T cell lineage large granular lymphocytosis associated with dasatinib therapy for Philadelphia chromosome positive leukemia. Haematologica. 2009;94(1):135-9.

5 Molldrem JJ, Lee PP, Wang C, Champlin RE, Davis MM. A PR1-human leukocyte antigen-A2 tetramer can be used to isolate low-frequency cytotoxic $\mathrm{T}$ lymphocytes from healthy donors that selectively lyse chronic myelogenous leukemia. Cancer Res. 1999;59(11):2675-81.

6 Schmitt M, Li L, Giannopoulos K, Chen J, Brunner C, Barth T, et al. Chronic myeloid leukemia cells express tumor-associated antigens eliciting specific CD8+ T-cell responses and are lacking costimulatory molecules. Exp Hematol. 2006;34(12):1709-19.

7 Bower H, Björkholm M, Dickman PW, Höglund M, Lambert PC, Andersson TM. Life expectancy of patients with chronic myeloid leukemia approaches the life expectancy of the general population. J Clin Oncol. 2016;34(24): 2851-7.

8 Corbin AS, Agarwal A, Loriaux M, Cortes J, Deininger MW, Druker BJ. Human chronic myeloid leukemia stem cells are insensitive to imatinib despite inhibition of BCR-ABL activity. J Clin Invest. 2011;121(1):396-409.

9 Hochhaus A, Baccarani M, Silver RT, Schiffer C, Apperley JF, Cervantes F, et al. European LeukemiaNet 2020 recommendations for treating chronic myeloid leukemia. Leukemia. 2020;34(4):966-84.

10 Jo T, Noguchi K, Hayashi S, Irie S, Hayase R, Shioya H, et al. Long-lasting memory of cellular immunity in a chronic myeloid leukemia patient maintains molecular response 5 after cessation of dasatinib. Oncol Lett. 2018;15(3):2935-8. 